\title{
Ethical issues in geriatric cranial neurosurgery
}

\author{
Laureen D. Hachem, MD, ${ }^{1}$ and Mark Bernstein, MD, MHSc ${ }^{1,2}$ \\ 'Division of Neurosurgery, Department of Surgery, University of Toronto; and 'Division of Neurosurgery, Toronto Western \\ Hospital, University Health Network, Toronto, Ontario, Canada
}

\begin{abstract}
The global demographic shift to an older population has led to the emergence of the new field of geriatric neurosurgery. Beyond the complexities of disease states and multimorbidity, advanced age brings with it intricate ethical issues pertaining to both the practice and provision of medical and surgical care. In this paper, the authors describe the central ethical themes seen across the spectrum of common neurosurgical conditions in the elderly and highlight the use of foundational ethical principles to help guide treatment decision-making.
\end{abstract}

https://thejns.org/doi/abs/10.3171/2020.7.FOCUS20447

KEYWORDS ethics; geriatric; neurosurgery; cranial; decision-making

$\mathrm{T}$ HE last decade has witnessed a shift in global demographics with a substantial increase in the proportion of older adults. It is estimated that the number of patients over the age of 65 years will double in the next 3 decades, with the "oldest old" (those older than 85 years) expected to triple during this time. ${ }^{1}$ The increase in our aging population has presented a number of challenges for the practice of modern medicine beyond just the complexities of disease states and multimorbidity. Advanced age brings with it intricate ethical issues pertaining to both the practice and provision of medical and surgical care.

These issues are well exemplified in a discipline such as neurosurgery, where diseases disproportionately affect the elderly, treatment-related risks are high, and outcomes may be poor regardless of intervention. Critical neurosurgical illness leaves patients unable to express their autonomy, the line between "benefit" and "risk" can be a fine one to walk in many situations, and the restraints of an overly burdened healthcare system demand consideration of the appropriate allocation of costly resources. The practicing neurosurgeon will need to navigate the ethical complexities inherent within the evolving field of geriatric neurosurgery. This paper outlines the main principles of bioethics and highlights how these underlie a number of key ethical challenges faced across the spectrum of neurosurgical conditions in elderly patients.

\section{Ethical Principles and Theories}

The foundation of medical ethics is built on four prin- ciple tenets: autonomy, beneficence, nonmaleficence, and justice. Each of these values has unique considerations in the context of geriatric neurosurgery. ${ }^{2}$

\begin{abstract}
Autonomy
While individuals have the autonomy to make their own treatment decisions, elderly patients faced with critical illness on a background of cognitive impairment may be unable to express their wishes. ${ }^{3}$ Surgeons must therefore make treatment decisions to best respect previously expressed goals of care. However, as most neurosurgical conditions arise unexpectedly, many individuals may not have considered end-of-life decisions and family members may be required to make hasty decisions between what appears to be "life" and "death."
\end{abstract}

\section{Beneficence and Nonmaleficence}

The principle of beneficence forms the foundation of medical and surgical care; i.e., one must do what will benefit the patient. Conversely, nonmaleficence requires that the physician act in a manner to avoid any harm to patients. High surgical risk on a background of medical complexity and poor prognosis among elderly patients has traditionally been thought to negate any minimal benefit from surgical intervention in many neurosurgical conditions. However, recent years have seen a paradigm shift away from viewing age alone as a cutoff for treatment, but instead incorporating functional/performance status into surgical decision-making. ${ }^{5}$ When considering the latter,

ABBREVIATIONS cSDH = chronic subdural hematoma. ACCOMPANYING EDITORIAL DOI: 10.3171/2020.7.FOCUS20667.

SUBMITTED May 29, 2020. ACCEPTED July 17, 2020.

INCLUDE WHEN CITING DOI: 10.3171/2020.7.FOCUS20447. 
the risk-benefit profile for many interventions has facilitated the selection of appropriate elderly patients who may, in fact, benefit from surgical intervention with a reasonable level of risk.

\section{Justice}

The constraints of an overly burdened healthcare system require physicians to triage patients to maximize treatment benefit. However, patients should never be denied surgery on the basis of age alone. Instead, factors such as frailty index and performance status must be incorporated into surgical decision-making.

The stewardship of medical resources has become an important topic of discussion, particularly in the setting of end-of-life care. It is estimated that close to $13 \%$ of total healthcare expenditure in the US is dedicated to patients in the last year of life, with the majority of these costs incurred in the final month of life. ${ }^{6-8}$ However, a major challenge arises in the fact that the "last month of life" can only be defined in retrospect and thus careful attention must be given to distinguish between resources put forth to save a life and those for end-of-life care. ${ }^{9}$ Moreover, some argue that high end-of-life care costs are, in part, a reflection of the broader issue of chronic disease burden, and thus interventions should focus on enhanced prevention and treatment of these conditions. ${ }^{8}$ Nevertheless, a concerted approach that focuses on clarifying patient goals of care, improving palliative care services, and enhancing home care supports will not only help better utilize healthcare resources, but also enhance the quality of care and comfort of elderly patients in their final months of life.

\section{Utilitarianism Versus Deontology}

In addition to the four primary ethical tenets, two main branches of ethical theory exist that influence decisionmaking: utilitarianism and deontology. ${ }^{10}$ Utilitarianism argues that one should act so as to produce the best outcome for the largest number of people. In contrast, deontology posits that one must do solely what is right for all people. The two perspectives are often viewed as a discord between society-centered versus patient-centered care. While most decisions in neurosurgery are based on a deontological framework, elements of utilitarianism have been incorporated into some aspects of treatment decision-making. This is well exemplified in cost-effectiveness analyses for elective spinal procedures in the elderly. ${ }^{11}$ Determining which patients maximally benefit from a specific intervention eliminates unnecessary procedures, therefore conserving valuable and finite resources.

\section{Ethical Themes in Geriatric Neurosurgery}

In applying the central ethical principles to the practice of geriatric neurosurgery, a number of overarching ethical themes arise, which are outlined in this section.

\section{Under-Access to Care}

Traditionally, elderly patients were viewed as uniformly less "fit" than younger patients and thus a less aggressive approach to treatment was commonly adopted. This un- der-access to care is a result of many contributory factors: poorer prognosis in many disease states, which can make treatment seem futile; high surgical risks in patients with less "stamina"; and patient preference valuing quality over quantity of life. While these are indeed valid arguments against the escalation of treatment, one must be careful not to stereotype based on age alone, but rather utilize an individualized approach that examines functional status and overall performance score when deciding whether to proceed with neurosurgical treatment.

\section{Over-Access to Care}

Recent advances in minimally invasive therapeutic strategies across neurosurgical subspecialties have introduced the moral quandary of over-access to care. The introduction of Gamma Knife surgery, endoscopic approaches, and endovascular techniques has provided surgeons with "lower-risk" treatment options for many neurosurgical conditions. Elderly patients who would otherwise be considered too high risk to undergo large craniotomies or open surgical procedures are now presented with alternative minimally invasive treatment modalities..$^{22}$ The central ethical issue here lies in whether these treatments, while having the appeal of lower risk or ease of operation, actually provide a meaningful clinical benefit to elderly patients and significantly improve prognosis or outcome.

\section{Futility}

The ethical tension between under- and over-access to care is rooted in the moral issue of treatment futility. The principle of beneficence would argue against any intervention in situations of clinical futility. But how do we define a futile treatment? Many would agree that an intervention that preserves unconsciousness without restoring any semblance of quality of life would be an effort in futility. ${ }^{13}$ However, who has the right to decide what is indeed futile: the physician, the patient, the substitute decision-maker? These challenging moral questions are further heightened in situations where the views of the treating physician and those of the family do not align. In an era of minimally invasive treatment options it is not uncommon for families to request "everything be done" to treat a relative out of fear of giving up when there is a seemingly low risk of intervention. However, while the upfront risk of a procedure may appear low, it is not negligible and may subsequently open the door to a series of downstream complications. These difficult situations must be guided by the principles of beneficence and nonmaleficence and demand careful reflection to the patient's long-term outlook.

\section{Consent}

To consent to a procedure, an individual must have the capacity to make one's own decisions. Specifically, they must demonstrate an understanding of their condition and the purpose of the proposed treatment, the associated risks and benefits, and the consequences of proceeding or abstaining from treatment. In most cases, capacity judgment is made by the treating physician and is generally implicit in our interactions with patients. The issue of capacity assessment in geriatric neurosurgery presents a 
number of ethical challenges. For example, not all patients with dementia should be assumed incapable of decisionmaking. Conversely, some patients with even very mild cognitive impairment who appear to be highly functioning with good performance scores on standard neurophysiological testing can have significant impairment in complex decision-making pertaining to their health. ${ }^{14,15}$ This underscores the importance of careful discussions with patients in addition to their families to truly gauge their understanding and treatment preferences.

In patients who are deemed unable to consent on their own, surgeons must rely on previously expressed wishes or substitute decision-makers to reach a treatment decision. Ethical issues arise when there is uncertainty on what a patient would have wanted or when substitute decision-makers act in line with their own values or interests rather than the patient's. Ultimately, it is the physician's moral duty to ensure that any decision be made in the best interest of the patient.

\section{Clinical Trials}

Clinical trials are central to the advancement of neurosurgery. However, many trials are conducted solely in younger populations, and even those focusing on geriatric cohorts typically include the "youngest of the old."16 Given the fact that elderly patients typically have more comorbidities and less functional reserve, it is reasonable to initially restrict experimental treatments to healthier patient cohorts. However, the exclusion of older patients from clinical trials can cause ethical challenges once a therapy is accepted into clinical practice. A lack of studies in geriatric patients makes clinicians hesitant to readily apply a novel treatment to this population. High functioning elderly patients may thus be denied potentially beneficial treatments on the basis of age alone.

The following sections describe the various ethical themes across the spectrum of common neurosurgical conditions in the elderly and highlight the use of the foundational ethical principles to help guide treatment decision-making.

\section{Functional Neurosurgery}

Functional neurosurgery is one of the most rapidly expanding surgical subspecialties, with an ongoing pipeline of new technologies to modulate the brain and growing indications to treat a wide array of neurological and psychiatric conditions. However, inherent within the development of novel therapies is a tendency to uniformly exclude older patients from clinical trials due to the perception that they are less fit than their younger counterparts. Indeed, trials examining new indications for deep brain stimulation or the efficacy of MR-guided focused ultrasound to deliver therapies across the blood-brain barrier generally include younger patient populations. ${ }^{17-19,48}$ The lack of evidence on the safety and efficacy of these novel therapies in geriatric patients can therefore limit their use within this population, leading to the moral inequality of treatment provision. While the exclusion of elderly patients from a clinical trial who are deemed medically or physically unfit for treatment is justified, careful attention should be given to incorporating other parameters, such as frailty indices and quality of life outcome measures, in order to capture older patients who could still benefit from a given therapy.

The application of functional neurosurgery to geriatric patients also highlights the ethical consideration of using surgery to alter the normal aging process. ${ }^{20}$ Currently, neuromodulation strategies are restricted to clear pathological symptoms such as the motor disturbances in Parkinson's disease or the cognitive and memory issues in Alzheimer's dementia. ${ }^{21}$ However, the line between pathological states and normal aging can be blurred as the concepts of health and disease evolve with time..$^{22,23}$ Incorporation of multidisciplinary assessment teams, comprehensive evaluations, and rigid diagnostic criteria is thus essential as checkpoints to prevent the use of novel technology to alter the course of normal aging.

\section{Neurooncology}

Malignant brain tumors are associated with worse survival in the elderly compared to younger patients. High-grade gliomas have a median overall survival of 4-6 months among patients $>65$ years old, compared to $12-14$ months in individuals $<70$ years old. ${ }^{24,25} \mathrm{~A}$ similar disparity in outcome is also reported for metastatic brain tumors. ${ }^{26}$

While aggressive treatment consisting of a combination of resection and chemoradiation is common in younger patients, concern regarding treatment-related toxicity and surgical risks among the elderly has traditionally led to undertreatment within this age group. However, growth in research pertaining to geriatric neurooncology has established tailored treatment regimens even among the oldest group of patients. ${ }^{27}$ Moreover, gross-total resection in select patients can still be associated with good outcomes and improved survival (Fig. 1). ${ }^{28,29}$ This has led to a shift away from considering age alone as a factor in determining treatment plans, but rather the concept of "physiological age." Treatment decision-making has now come to rely more on functional status and performance scores to select elderly patients who may achieve comparable outcomes to younger patients.

The introduction of less-invasive treatment options for brain tumors such as Gamma Knife surgery or laser interstitial thermal therapy has further expanded treatment options for elderly patients. However, one can only wonder whether this has further tipped the scales toward overtreating patients in some situations. It is important to define the ethical lines in terms of our current technological capabilities. Indeed, as the old adage states, "just because we can doesn't mean we should." An 83-year-old patient with congestive heart failure, metastatic lung cancer, and a 3 -cm vestibular schwannoma will gain little clinical benefit from Gamma Knife surgery to the lesion, despite the therapy's relatively low risk and good tumor control rate. Surgeons must be cognizant of their own potential biases as studies have found that physicians tend to overestimate prognosis and treatment-related benefit. ${ }^{30}$ As we continue to push the limits of surgical innovation with the appeal of minimally invasive lower-risk therapies, it is imperative that we continue to utilize evidence-based practices 

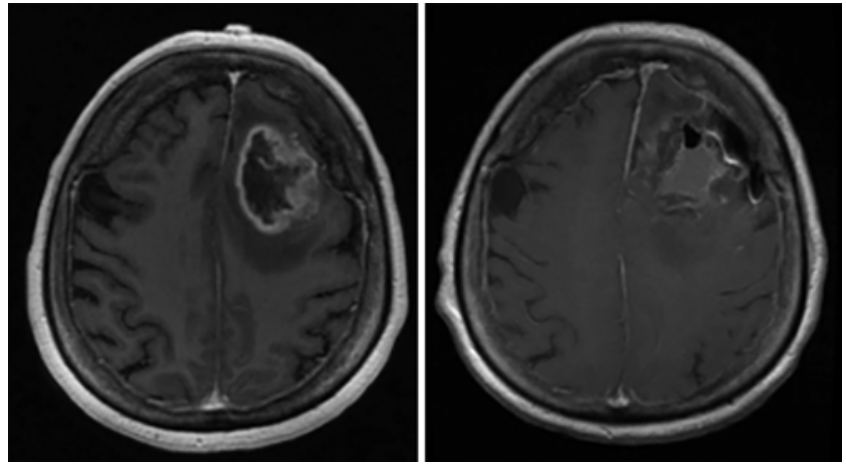

FIG. 1. Pre- (left) and postoperative (right) axial MR images from an 82-year-old man who underwent a left craniotomy for gross-total resection of a left frontal glioblastoma.

in treatment decision-making. Moreover, incorporating individual patient priorities and unique experiences with terminal disease into treatment-related discussions can further facilitate the establishment of an optimal management plan. ${ }^{31}$

Ethical issues with clinical trial design also apply to the field of geriatric neurooncology. Novel targeted biological agents and viral-based drug delivery platforms are on the horizon for clinical translation, but studies on their efficacy are typically conducted in younger cohorts. Moreover, many of these therapies carry a high cost, which, together with the paucity of clinical evidence to support their use in an older age group, can lead to the exclusion of geriatric patients from potentially beneficial therapies.

\section{Neurotrauma \\ Chronic Subdural Hematoma}

Chronic subdural hematoma (cSDH) is largely a disease of the elderly, with a rising incidence due to an aging population prone to falls and high rates of anticoagulant usage $^{32,33}$ In some cases, management is straightforward: a small asymptomatic cSDH can be monitored serially, whereas a large bleed with significant mass effect and progressive weakness requires surgical evacuation (Fig. 2). However, many cases are equivocal. Take, for example, the scenario of an 87-year-old man with extensive cardiac history, receiving anticoagulant therapy for a mechanical heart valve, who has a $1.5-\mathrm{cm}$ left $\mathrm{cSDH}$ presenting with 2 months of slight instability in his gait but otherwise is fully functional. Surgical management would almost certainly be recommended in a younger individual with fewer medical comorbidities given the impact on their function and the risk of further mass effect in a less atrophic brain. In elderly patients, however, the risk-benefit profile of surgery is often less binary, and the surgeon must balance the ethical tension between over- and undertreatment.

Recurrence rates of cSDH are reported as high as 30\% following surgical evacuation, ${ }^{34,35}$ with increased age an independent predictor of unfavorable outcome. ${ }^{36}$ Furthermore, many conditions, such as cervical myelopathy or dementia, that are common in the elderly population can mimic symptoms of cSDH. As such, evacuation of the bleed may not improve preoperative symptoms and a con-
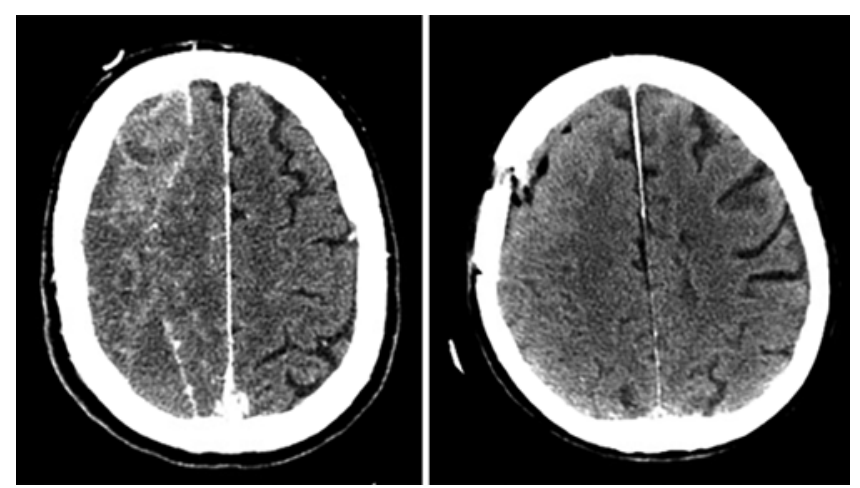

FIG. 2. Pre- (left) and postoperative (right) axial CT scans in a 75-yearold man who underwent a right mini-craniotomy for subdural hematoma evacuation.

servative approach may be justified. Conversely, one could argue that burr hole or minicraniotomy drainage of cSDH is a small operation made further safe by the advent of awake/neuroleptic anesthesia, which avoids the risks associated with a general anesthetic. The benefits of evacuating the bleed may therefore justify surgical management given the seemingly low risk of intervention. The decision is made further complicated in elderly patients with recurrent bleeds despite multiple previous surgeries. In these cases, the surgeon may find it difficult to rationalize further surgical intervention given the track record of futility and therefore justify a conservative approach. Middle meningeal artery embolization has emerged as a potential minimally invasive alternative to surgery for recurrent cSDH, with a number of studies demonstrating its efficacy in reducing hemorrhage recurrence. ${ }^{4}$ However, this strategy does not address the primary issue of mass effect that is commonly seen in patients, and further clinical study is required to support its efficacy and safety, particularly among the elderly. ${ }^{37}$

While there are no guidelines to aid in approaching the difficult treatment decisions associated with managing cSDH in the elderly, nor can they supplant good clinical judgment, an awareness of the ethical issues inherent in these cases is important when deciding on a management plan.

\section{Traumatic Brain Injury}

The morbidity associated with traumatic brain injury in the elderly is high, often leading to permanent physical and cognitive disability. ${ }^{38}$ Surgical treatment in the form of external ventricular drainage, clot evacuation, or decompressive craniectomy is offered in cases of significant mass effect or brain herniation in which patients are not past the point of salvage. The central ethical issue here is one of futility: would surgical intervention improve outcomes or lead to meaningful survival? An essential component in answering this question involves determining an accurate assessment of prognosis. Families want to know how long their loved one will live, whether they will ever regain the ability to breathe or eat independently, or even recognize their own relatives. These questions are particularly challenging to answer in geriatric patients as 
standard assessment tools and prediction models for survival and prognosis used in younger cohorts of patients with traumatic brain injury appear to have less predictive value in the elderly. ${ }^{38,39}$ Specifically, measures of frailty, multimorbidity, or polypharmacy are typically excluded, as are important outcomes such as quality of life. ${ }^{40}$ While most surgeons will have a reasonable prediction of prognosis, in many cases there will be an irreducible level of uncertainty. It is essential that these potential uncertainties be articulated to the family when arriving at a treatment plan. Even with a reasonable prediction on prognosis, the concept of "meaningful" survival can be a source of further contention. While many would agree that loss of independence and reliance on intensive care unit support for survival would not be a meaningful way to live, some substitute decision-makers are unable to make the decision to "pull the trigger" on a relative. These conflicts demand open and clear conversations between surgeons, intensivists, and a patient's family or substitute decisionmaker.

\section{Vascular Neurosurgery}

Malignant middle cerebral artery infarction is a devastating condition with high associated morbidity. A series of foundational randomized controlled trials provided strong evidence that decompressive hemicraniectomy reduces mortality in patients 60 years of age or younger with favorable outcomes. ${ }^{41-44}$ The benefits in the elderly, however, have remained controversial due to both a paucity of data within this group and the associated ethical implications. While a follow-up randomized controlled trial demonstrated increased survival in patients undergoing decompressive craniectomy over the age of 60 years ${ }^{45}$ patients with significant medical comorbidities were excluded, thus raising concerns on the generalizability to the "average" geriatric population. Moreover, this increase in survival was associated with a significant level of disability.

The moral dilemma in this situation arises from the question of what constitutes an acceptable outcome. Few individuals would accept survival having lost all independence and quality of life. ${ }^{46}$ While studies have shown that patients who underwent decompressive craniectomy would retrospectively have consented to the same procedure knowing the functional state they would be in, ${ }^{47}$ one can only question whether patients have simply adapted to their current situation. Moreover, it is possible that the surgeon's views are in conflict with those of family members who might hold survival above all else, regardless of quality of life. It is of utmost importance to clearly outline the degree of impairment that could be expected after surgery to ensure that families can make an accurate assessment on whether this would be an acceptable outcome for the patient. Nevertheless, it is the physician's responsibility to ensure that decisions are made to benefit the patient and limit harm. In situations where surgery will not improve survival or will result in a significant amount of disability and permanent dependence on intensive care unit-level care, physicians may not offer surgery upfront given the potential harm the patient would endure.

\section{Conclusions}

The global demographic shift to an older population has led to the emergence of the new field of geriatric neurosurgery. Having a clear ethical framework and knowledge of the ethical issues inherent within this field will be of utmost importance in delivering the highest level of care to the unique population of geriatric neurosurgical patients.

\section{References}

1. World Report on Ageing and Health. World Health Organization; 2015. Accessed August 6, 2020. https://www.who.int/ ageing/events/world-report-2015-launch/en/

2. Mueller PS, Hook CC, Fleming KC. Ethical issues in geriatrics: a guide for clinicians. Mayo Clin Proc. 2004;79(4):554562.

3. Terranova C, Cardin F, Pietra LD, et al. Ethical and medicolegal implications of capacity of patients in geriatric surgery. Med Sci Law. 2013;53(3):166-171.

4. Lukovits TG, Bernat JL. Ethical approach to surrogate consent for hemicraniectomy in older patients with extensive middle cerebral artery stroke. Stroke. 2014;45(9):2833-2835.

5. Bligh ER, Sinha P, Smith D, Al-Tamimi YZ. Thirty-day mortality and survival in elderly patients undergoing neurosurgery. World Neurosurg. 2020;133:e646-e652.

6. Lubitz JD, Riley GF. Trends in Medicare payments in the last year of life. N Engl J Med. 1993;328(15):1092-1096.

7. Aldridge MD, Kelley AS. The myth regarding the high cost of end-of-life care. Am J Public Health. 2015;105(12):24112415.

8. French EB, McCauley J, Aragon M, et al. End-of-life medical spending in last twelve months of life is lower than previously reported. Health Aff (Millwood). 2017;36(7):1211-1217.

9. Emanuel EJ, Emanuel LL. The economics of dying. The illusion of cost savings at the end of life. $N$ Engl J Med. 1994;330(8):540-544.

10. Mandal J, Ponnambath DK, Parija SC. Utilitarian and deontological ethics in medicine. Trop Parasitol. 2016;6(1):5-7.

11. Adogwa O, Owens R, Karikari I, et al. Revision lumbar surgery in elderly patients with symptomatic pseudarthrosis, adjacent-segment disease, or same-level recurrent stenosis. Part 2. A cost-effectiveness analysis: clinical article. J Neurosurg Spine. 2013;18(2):147-153.

12. Chibbaro S, Di Rocco F, Makiese O, et al. Neurosurgery and elderly: analysis through the years. Neurosurg Rev. 2010;34(2):229-234.

13. Aghabarary M, Dehghan Nayeri N. Medical futility and its challenges: a review study. J Med Ethics Hist Med. 2016;9:11.

14. Marson DC, Ingram KK, Cody HA, Harrell LE. Assessing the competency of patients with Alzheimer's disease under different legal standards. A prototype instrument. Arch Neurol. 1995;52(10):949-954.

15. Manes F, Torralva T, Ibáñez A, et al. Decision-making in frontotemporal dementia: clinical, theoretical and legal implications. Dement Geriatr Cogn Disord. 2011;32(1):11-17.

16. Ilgili $\mathrm{O}$, Arda B, Munir K. Ethics in geriatric medicine research. Turk Geriatri Derg. 2014;17(2):188-195.

17. Holtzheimer PE, Husain MM, Lisanby SH, et al. Subcallosal cingulate deep brain stimulation for treatment-resistant depression: a multisite, randomised, sham-controlled trial. Lancet Psychiatry. 2017;4(11):839-849.

18. Rodriguez-Oroz MC, Obeso JA, Lang AE, et al. Bilateral deep brain stimulation in Parkinson's disease: a multicentre study with 4 years follow-up. Brain. 2005;128(Pt 10):22402249.

19. Park SH, Kim MJ, Jung HH, et al. Safety and feasibility of 
multiple blood-brain barrier disruptions for the treatment of glioblastoma in patients undergoing standard adjuvant chemotherapy. J Neurosurg. Published online January 3, 2020. doi:10.3171/2019.10.JNS192206

20. Mendelsohn D, Lipsman N, Bernstein M. Neurosurgeons' perspectives on psychosurgery and neuroenhancement: a qualitative study at one center. J Neurosurg. 2010;113(6):1212-1218.

21. Laxton AW, Tang-Wai DF, McAndrews MP, et al. A phase I trial of deep brain stimulation of memory circuits in Alzheimer's disease. Ann Neurol. 2010;68(4):521-534.

22. Lipsman N, Lozano AM. Cosmetic neurosurgery, ethics, and enhancement. Lancet Psychiatry. 2015;2(7):585-586.

23. Lipsman N, Zener R, Bernstein M. Personal identity, enhancement and neurosurgery: a qualitative study in applied neuroethics. Bioethics. 2009;23(6):375-383.

24. Iwamoto FM, Reiner AS, Panageas KS, et al. Patterns of care in elderly glioblastoma patients. Ann Neurol. 2008;64(6):628-634.

25. Stupp R, Mason WP, van den Bent MJ, et al. Radiotherapy plus concomitant and adjuvant temozolomide for glioblastoma. N Engl J Med. 2005;352(10):987-996.

26. Salcman M. Brain tumors and the geriatric patient. $J$ Am Geriatr Soc. 1982;30(8):501-508.

27. Mansouri A, Hachem LD, Mansouri S, et al. MGMT promoter methylation status testing to guide therapy for glioblastoma: refining the approach based on emerging evidence and current challenges. Neuro Oncol. 2019;21(2):167-178.

28. Moraes FY, Lo A, Morgan ER, et al. Management and outcomes in the oldest-old population with glioblastoma. Can J Neurol Sci. 2018;45(2):199-205.

29. Noorbakhsh A, Tang JA, Marcus LP, et al. Gross-total resection outcomes in an elderly population with glioblastoma: a SEER-based analysis. J Neurosurg. 2014;120(1):31-39.

30. Glare P, Virik K, Jones M, et al. A systematic review of physicians' survival predictions in terminally ill cancer patients. BMJ. 2003;327(7408):195-198.

31. Lipsman N, Skanda A, Kimmelman J, Bernstein M. The attitudes of brain cancer patients and their caregivers towards death and dying: a qualitative study. BMC Palliat Care. 2007;6:7.

32. Yang W, Huang J. Chronic subdural hematoma: epidemiology and natural history. Neurosurg Clin N Am. 2017;28(2):205-210.

33. Balser D, Farooq S, Mehmood T, et al. Actual and projected incidence rates for chronic subdural hematomas in United States Veterans Administration and civilian populations. $J$ Neurosurg. 2015;123(5):1209-1215.

34. Ducruet AF, Grobelny BT, Zacharia BE, et al. The surgical management of chronic subdural hematoma. Neurosurg Rev. 2012;35(2):155-169.

35. Nakaguchi H, Tanishima T, Yoshimasu N. Factors in the natural history of chronic subdural hematomas that influence their postoperative recurrence. J Neurosurg. 2001;95(2):256262.

36. Brennan PM, Kolias AG, Joannides AJ, et al. The management and outcome for patients with chronic subdural hematoma: a prospective, multicenter, observational cohort study in the United Kingdom. J Neurosurg. 2017;127(4):732-739.

37. Haldrup M, Ketharanathan B, Debrabant B, et al. Embolization of the middle meningeal artery in patients with chronic subdural hematoma-a systematic review and meta-analysis. Acta Neurochir(Wien). 2020;162(4):777-784.
38. Gardner RC, Dams-O’Connor K, Morrissey MR, Manley GT. Geriatric traumatic brain injury: epidemiology, outcomes, knowledge gaps, and future directions. J Neurotrauma. 2018;35(7):889-906.

39. Salottolo K, Levy AS, Slone DS, et al. The effect of age on Glasgow Coma Scale score in patients with traumatic brain injury. JAMA Surg. 2014;149(7):727-734.

40. Joseph B, Pandit V, Zangbar B, et al. Superiority of frailty over age in predicting outcomes among geriatric trauma patients: a prospective analysis. JAMA Surg. 2014;149(8):766772 .

41. Geurts M, van der Worp HB, Kappelle LJ, et al. Surgical decompression for space-occupying cerebral infarction: outcomes at 3 years in the randomized HAMLET trial. Stroke. 2013;44(9):2506-2508.

42. Hofmeijer J, Kappelle LJ, Algra A, et al. Surgical decompression for space-occupying cerebral infarction (the Hemicraniectomy After Middle Cerebral Artery infarction with Lifethreatening Edema Trial [HAMLET]): a multicentre, open, randomised trial. Lancet Neurol. 2009;8(4):326-333.

43. Jüttler E, Schwab S, Schmiedek P, et al. Decompressive Surgery for the Treatment of Malignant Infarction of the Middle Cerebral Artery (DESTINY): a randomized, controlled trial. Stroke. 2007;38(9):2518-2525.

44. Vahedi K, Vicaut E, Mateo J, et al. Sequential-design, multicenter, randomized, controlled trial of early decompressive craniectomy in malignant middle cerebral artery infarction (DECIMAL Trial). Stroke. 2007;38(9):2506-2517.

45. Jüttler E, Unterberg A, Woitzik J, et al. Hemicraniectomy in older patients with extensive middle-cerebral-artery stroke. $N$ Engl J Med. 2014;370(12):1091-1100.

46. Unterhofer C, Ho WM, Wittlinger K, et al. "I am not afraid of death"-a survey on preferences concerning neurosurgical interventions among patients over 75 years. Acta Neurochir (Wien). 2017;159(8):1547-1552.

47. Kiphuth IC, Köhrmann M, Lichy C, et al. Hemicraniectomy for malignant middle cerebral artery infarction: retrospective consent to decompressive surgery depends on functional long-term outcome. Neurocrit Care. 2010;13(3):380-384.

48. Mainprize T, Lipsman N, Huang Y, et al. Blood-brain barrier opening in primary brain tumors with non-invasive MRguided focused ultrasound: a clinical safety and feasibility study. Sci Rep. 2019;9(1):321.

\section{Disclosures}

The authors report no conflict of interest concerning the materials or methods used in this study or the findings specified in this paper.

\section{Author Contributions}

Conception and design: both authors. Drafting the article: both authors. Critically revising the article: both authors. Reviewed submitted version of manuscript: both authors. Approved the final version of the manuscript on behalf of both authors: Bernstein.

\section{Correspondence}

Mark Bernstein: Toronto Western Hospital, Toronto, ON, Canada. mark.bernstein@uhn.ca. 